\title{
An Explanation for Pulsar Mode Changing Phenomenon
}

\author{
B. Zhang ${ }^{1}$, G.J. Qiao ${ }^{1}$, W.P. $\operatorname{Lin}^{1,2}$, J.L. $\operatorname{Han}^{2}$ \\ 1. Department of Geophysics, Peking University, Beijing 100871, China \\ 2. Beijing Astronomical Observatory, CAS, Beijing 100080, China
}

\begin{abstract}
There are three mechanisms to cause pulsar inner gap breakdown: the inverse Compton scattering (ICS) of the high energy particles off the thermal-peak photons, off the resonant-frequency photons and the curvature radiation (CR). The pulsar mode-changing phenomenon can be interpreted as a switching effect between the resonant ICS sparking mode and the thermal ICS sparking mode.
\end{abstract}

Mode changing is a strange phenomenon found in some pulsars. The integrated pulse profiles of some pulsars will switch suddenly to the so-called "abnormal" mode, staying in the state for a period of time, and then switch back again to the "normal" mode. The switching time scale is very short, less than one pulsar period. Usually, mode-changing behaviour can be observed at almost all wave bands. No acceptable explanation of the phenomenon has yet been proposed.

The inner gap model (Ruderman \& Sutherland 1975, hereafter RS75) can be improved by taking into account the ICS process involving high-energy particles and thermal photons near the neutron star, which usually produces much more energetic $\gamma$-rays, and hence becomes the dominant mechanism of inner gap sparking (Zhang \& Qiao 1995, hereafter paper I; Qiao \& Zhang 1995, hereafter paper II).

Usually, the gap properties are determined under the assumption $l \sim h$, where $l$ is the mean free path of the $\gamma$-rays in $\gamma-B$ process, and $h$ is the gap height (RS75; paper I; paper II). The photon free path in a strong magnetic field is (Erber 1966): $l=\frac{4.4}{e^{2} / \hbar c} \frac{\hbar}{m_{e} c} \frac{B_{q}}{B_{\perp}} \exp \left(\frac{4}{3 \chi}\right)$ for $\chi \ll 1$, where $\chi \equiv \frac{E_{\gamma}}{2 m_{e} c^{2}} \sin \theta \frac{B}{B_{q}}=$ $\frac{E_{\gamma}}{2 m_{e} c^{2}} \frac{B_{\perp}}{B_{q}}$ and $B_{\perp}=\left(B_{z}^{2}+B^{2} \frac{(\boldsymbol{\Omega} \times \mathbf{r})^{2}}{c^{2}}\right)^{1 / 2}$ (Daugherty \& Lerche 1975; Paper II). Here $e$ is the electric charge, $m_{e}$ the mass of an electron, $c$ the light speed, $\hbar$ the Planck's constant, $B_{q}=4.414 \times 10^{13} G$ the critical magnetic field, $E_{\gamma}$ the energy of $\gamma$-rays, $\theta$ the pitch angle of the $\gamma$-photons to the magnetic fields, $\Omega=2 \pi / P$ the angular frequency for pulsar period $P, B$ the magnetic field at a distance $r$ from the star center, $B_{z}$ the perpendicular magnetic component with respect to the direction of the $\gamma$-photon in the corotating frame.

The potential across the inner gap (for $h \ll r_{p}, \mathrm{RS75}$ ) is $\Delta V=\frac{\Omega B}{c} h^{2}$, where $r_{p} \sim R\left(\frac{\Omega R}{c}\right)^{1 / 2}$ is the polar cap radius. The maximum Lorentz factor of the particles gained from this potential is $\gamma=\frac{e \Delta V}{m_{e} c^{2}}$.

The above equations are sufficient to describe the inner gap properties once the energy of the $\gamma$-ray $E_{\gamma}$ is determined. Different $E_{\gamma}$ will natually result in different gap properties. There are three prefered possibilities for $E_{\gamma}$ : (1). The 
thermal ICS sparking case (see Paper II): $E_{\gamma} \simeq \gamma^{2} \hbar \omega_{m} \simeq 2.82 \gamma^{2} k T$ where $\omega_{m}$ is the peak-frequency of thermal radiation at temperature $T, k$ is the Boltzman constant; (2). The resonant ICS sparking case (see Paper I): $E_{\gamma} \simeq \gamma^{2} \hbar \omega_{\text {res }}=$ $\gamma \hbar \frac{e B}{m_{e} c}$, where $\omega_{\text {res }}$ is the frequency at which the ICS cross section is greatly enhanced (resonant frequency); (3). The $C R$ sparking case: $E_{\gamma} \simeq \hbar \omega_{c r}=$ $\hbar \frac{3}{2} \gamma^{3} c / \rho$, where $\omega_{c r}$ is the characteristic frequency for curvature radiation.

Three criteria can be used to determine which process dominates: (1). The larger $E_{\gamma \perp}$ will more likely cause the gap breakdown; (2). The potential drop across the gap should be smaller than the maximum potential of a rotating neutron star $\Delta V_{\max }=\frac{\Omega B}{2 c} r_{p}^{2}$ due to homopolar effect; (3). The mean free path of the particles to produce a certain $\gamma$-ray photon for gap breakdown should be smaller than the gap height.

According to calculations, we find that the mean free paths of the particles are quite sensitive to the surface temperature. Since criterion (2) can usually be satisfied, examining criterion (3) and (1), we get two critical temperatures of gap sparking: $T_{01}$, above which the gap can break down via thermal ICS; and $T_{02}$, above which the gap can break down via resonant ICS. Only when $T<\min \left(T_{01}, T_{02}\right)$, will the CR process be dominant.

Thus three possible modes could cause gap breakdown, namely, the thermal ICS mode, the resonant ICS mode and the CR mode. Which one dominates depends on the actual temperature of a neutron star. The temperature could fluctuate around a certain temperature $T$ due to various reasons (e.g. the starquake, hitting by the high energy particles, etc.). If it goes across one of the critical temperatures, the gap sparking mechanism will change suddenlly.

Pulsar mode-changing phenomenon can be interpreted as the switching effect between the resonant ICS sparking mode and the thermal ICS sparking mode. (1). We found a preferred region in the diagram for mode-changing, where the critical temperatures $T_{01}$ are around the actual value of the surface temperatures of the neutron stars. The observed mode-changing pulsars just lie in this prefered region. (2). Our mechanism can interpret the short time scale of the mode-changing, because the critical temperature presents a switching effect. (3). Different sparking mechanisms can result in different gap properties (e.g. the acceleration potential, the Lorentz factors of the escaping particles, the gap height, etc.), and hence result in different radio emission beams.

Acknowledgments. This work is supported in part by NNSF of China, the Climbing Project and Doctoral Program Foundation of Institution of Higher Education in China.

\section{References}

Daugherty J.K., Lerche I., 1975, Ap\&SS 38, 437

Erber J., 1966, Rev.Mod.Phys. 38, 626

Qiao G.J., Zhang B., 1995, A\&A 306, L5 (Paper 2)

Ruderman M.A., Sutherland P.G., 1975, ApJ 196, 51

Zhang B., Qiao G.J., 1995, A\&A, in press (Paper 1) 